\title{
Quantum Communication Complexity of Distribution Testing
}

\author{
Aleksandrs Belovs ${ }^{1}$, Arturo Castellanos ${ }^{2}$, François Le Gall ${ }^{3}$, \\ Guillaume Malod ${ }^{4}$, Alexander A. Sherstov ${ }^{5}$ \\ ${ }^{1}$ University of Latvia \\ ${ }^{2}$ Kyoto University \\ ${ }^{3}$ Nagoya University \\ ${ }^{4}$ Université de Paris \\ ${ }^{5}$ University of California, Los Angeles
}

June 29, 2020

\begin{abstract}
The classical communication complexity of testing closeness of discrete distributions has recently been studied by Andoni, Malkin and Nosatzki (ICALP'19). In this problem, two players each receive $t$ samples from one distribution over $[n]$, and the goal is to decide whether their two distributions are equal, or are $\epsilon$-far apart in the $l_{1}$-distance. In the present paper we show that the quantum communication complexity of this problem is $\tilde{O}\left(n /\left(t \epsilon^{2}\right)\right)$ qubits when the distributions have low $l_{2}$-norm, which gives a quadratic improvement over the classical communication complexity obtained by Andoni, Malkin and Nosatzki. We also obtain a matching lower bound by using the pattern matrix method. Let us stress that the samples received by each of the parties are classical, and it is only communication between them that is quantum. Our results thus give one setting where quantum protocols overcome classical protocols for a testing problem with purely classical samples.
\end{abstract}

Keywords - Quantum communication complexity, Distribution testing, Lower bounds

\section{Introduction}

Background. Property testing [Gol17, GR11] is the task of (approximately) distinguishing objects having some specific property from those which are "far" from having it, without necessarily looking at the objects in their entirety. An interesting subfield is discrete distribution testing [Can15], where the objects are probability distributions.

One of the main tasks in (discrete) distribution testing, namely closeness testing, is about deciding whether two distributions $p$ and $q$ over $[n]$ are equal or $\epsilon$-far from each other in the $l_{1}$-norm, given access only to a limited number of samples of each distribution. Early testers $\mathrm{BFR}^{+} 00, \mathrm{BFR}^{+} 13$ used a method based on collisions. Using improved estimators, testers with optimal sample complexity have then been constructed CDVV14, DK16.

Very recently, Andoni, Malkin and Nosatzki [AMN19] have, for the first time, considered distribution testing in the two-party setting. Here two players, Alice and Bob, each own as input $t$ samples of the distributions $p$ and $q$ : Alice has $t$ samples from $p$ and Bob has $t$ samples from $q$. The goal is for Alice and Bob to decide if the two distributions are equal or $\epsilon$-far from each other in the $l_{1}$-norm, using as little communication as possible. By adapting the techniques from prior works [CDVV14, DK16, Andoni, Malkin and Nosatzki have shown that this problem (named $2 \mathrm{PCT}_{n, t, \epsilon}$ in [AMN19]) can be solved with high probability using $O\left(\frac{n^{2}}{t^{2} \epsilon^{4}}+1\right)$ bits of communication whenever $t$ is above the information-theoretic lower bound (given in Equation (11) below) which is the minimum number of samples needed so that meaningful information about $p$ and $q$ can be extracted from them. They also showed a matching lower bound $\Omega\left(\frac{n^{2}}{t^{2}}\right)$ on the two-party communication complexity of $2 \mathrm{PCT}_{n, t, 1 / 2}$. 
Our results. In this paper we investigate the quantum communication complexity of this problem. Our main result shows that a significant advantage can be obtained in the quantum setting when at least one of the two distributions has low $l_{2}$-norm. Concretely, for any $\gamma>0$, we consider the version of $2 \mathrm{PCT}_{n, t, \epsilon}$ in which the inputs $p, q$ satisfy the condition $\min \left(\|p\|_{2},\|q\|_{2}\right) \leq \gamma t \epsilon^{2} / n$. We denote this problem 2PCT ${ }_{n, t, \epsilon}^{\gamma}$. The lower bound from [AMN19] shows that for $\gamma=\Omega\left(\frac{1}{\sqrt{\log n}}\right)$, this version is as hard as the original version of the problem 1 As in all previous works [AMN19, CDVV14, DK16, we will assume throughout the paper that $t$ is above the information-theoretic threshold:

$$
t \geq C \max \left(n^{2 / 3} \cdot \epsilon^{-4 / 3}, \sqrt{n} \cdot \epsilon^{-2}\right),
$$

where $C$ is a universal constant (see AMN19, CDVV14 for details).

We first show the following theorem.

Theorem 1. There exists an absolute constant $\gamma_{0}$ such that the following holds: for all $\gamma \leq \gamma_{0}$, the problem $2 \mathrm{PCT}_{n, t, \epsilon}^{\gamma}$ can be solved with high probability by a quantum protocol that uses $\tilde{O}\left(\frac{n}{t \epsilon^{2}}+1\right)$ qubits of communication.

Theorem 1 shows that a significant advantage (a quadratic improvement in the communication complexity) can be obtained in the quantum setting when at least one of the two distributions has low $l_{2}$-norm.

We also obtain the following lower bound, which shows that the upper bound of Theorem 1 is optimal, even for $\epsilon=1 / 2$.

Theorem 2. There exists an absolute constant $c>1$ such that the following statement holds for any value $t \leq n / \log ^{c} n$ and any $\gamma=\Omega(1 / \sqrt{\log n})$ : any quantum protocol that solves $2 \mathrm{PCT}_{n, t, 1 / 2}^{\gamma}$ with high probability requires $\tilde{\Omega}(n / t)$ qubits of communication.

Precisely, the lower bound holds for any $\gamma$ greater than some $\gamma_{L W}=O\left(\frac{1}{\sqrt{\log n}}\right)$. Therefore there is a regime where $\gamma_{L W} \leq \gamma_{0}$, for which our upper bound is then tight.

Overview of our main techniques. Our upper bound (Theorem 1) is obtained by following the framework used in AMN19, which relies on the estimator from CDVV14 for the $l_{2}$-distance. Indeed, as suggested by $\mathrm{BFR}^{+} 00$, CDVV14 and then extensively studied in [DK16, there is a reduction from closeness testing in the $l_{1}$-distance to closeness testing in the $l_{2}$-distance. The efficiency of the reduction, however, depends on the $l_{2}$-norm of the distribution. The protocol in AMN19] thus proceeds in two steps. In the first step, Bob shares some information with Alice about the observed shape of his distribution, so that they can recast their distributions into two distributions $p^{\prime}$ and $q^{\prime}$ that have smaller $l_{2}$-norm while preserving the $l_{1}$-distance (i.e., $\left\|p^{\prime}-q^{\prime}\right\|_{1}=\|p-q\|_{1}$ ). In the second step, Alice and Bob use the reduction to closeness testing in the $l_{2}$-distance mentioned and implement the estimator of CDVV14] in the two-party setting. This estimator requires estimating with good precision the $l_{2}$-distance between two vectors. This is done by using a two-party implementation of the sketching method by Alon, Matias and Szegedy AMS99.

For the case of low-norm distribution (more precisely, when considering the problem $2 \mathrm{PCT}_{n, t, \epsilon}^{\gamma}$ with $\gamma$ constant), the first step is unnecessary: the two distributions already have a low enough $l_{2}$-norm, so that the reduction to closeness testing in the $l_{2}$-distance can be used without any preprocessing. We thus only need to show how to implement the second step from AMN19 more efficiently using quantum communication. The key idea is to use the quantum algorithm by Montanaro [Mon16] which gives a quadratic speedup over the classical sketching method from AMS99] in the query complexity model. We show how to adapt this quantum algorithm to the two-party setting in Section 3.2 .

Our lower bound (Theorem 2) first applies the same reduction as in AMN19, which reduces some specific version of the Gap-Hamming distance to $2 \mathrm{PCT}_{n, t, 1 / 2}^{\gamma}$. In the classical case, AMN19 showed that the communication complexity of that version of the Gap-Hamming distance is $\tilde{\Omega}\left((n / t)^{2}\right)$ bits. Our main technical contribution proves that the quantum communication complexity of this problem is $\tilde{\Omega}(n / t)$. We use the pattern matrix method She11. To obtain our lower bound, we show that the pattern matrix method, which is generally formulated only for total functions, can be generalized to partial functions.

\footnotetext{
${ }^{1}$ Indeed, the lower bound $\Omega\left(\frac{n^{2}}{t^{2}}\right)$ from [AMN19] is shown for input distributions such that $\|p\|_{2}=\|q\|_{2}=$ $O\left(\frac{t \epsilon^{2}}{n \sqrt{\log n}}\right)$.
} 
Relation with known quantum advantages in testing and learning. Several quantum algorithms have been designed for property testing and learning theory (we refer to [AdW17] and MdW16. for excellent surveys of these fields). In most settings considered so far in quantum learning theory, however, the quantum algorithms crucially exploit the fact that the data can be accessed in a quantum way (e.g., we can query a quantum superposition of samples), which makes it difficult to directly compare the performance of quantum algorithms with the performance of classical methods (which can only access the data in a classical way). The results of the present paper show a quantum advantage, in terms of communication cost, for the setting where both classical and quantum protocols can access the data in the same way - the input is given as a set of classical samples.

Open problem. An intriguing question is whether a similar quantum advantage is achievable when both input distributions have higher $l_{2}$-norm, i.e., whether the upper bound we obtain in Theorem 1 holds not only for constant $\gamma$ but also for larger values of $\gamma$. Currently, we do not know how to improve the complexity of the first part of the classical protocol from AMN19, which (as mentioned above) converts the input distributions into distributions of sufficiently small $\ell_{2}$-norm, using quantum communication. We left this question as an open problem.

\section{Preliminaries}

\subsection{Definitions and Notations}

A typical communication task for Alice and Bob is to compute (sometimes only with some probability of success) a function $f$ on some inputs $x, y$ where $x$ is given to Alice and $y$ to Bob. A communication protocol is an algorithmic description of message sending between Alice and Bob that solves the task for any possible pair of inputs. The communication complexity [KN97] of such function is the minimum required numbers of bits the most efficient protocol solving the task must exchange in the worst case (regarding inputs).

The quantum communication complexity dW02, Bra04 of a function is the equivalent using qubits instead of bits. Qubits correspond to elements of some Hilbert space of dimension 2. We will use the bra-ket notation $|\phi\rangle_{R}$ to denote a qubit (and by extension an $n$-qubit string) $\phi$ of a register $R$.

As described in the introduction, Alice's input consists of $t$ samples from a discrete distribution $p:[n] \rightarrow(0,1)$. Bob's input consists of $t$ samples from a discrete distribution $q:[n] \rightarrow(0,1)$. We call $X_{i}$ (resp. $Y_{i}$ ) the number of samples of Alice (resp. Bob) corresponding to element $i$. We call $X, Y \in[t]^{n}$ the occurrence vectors of Alice and Bob, i.e., the vectors with $i$-th coordinate $X_{i}$ and $Y_{i}$, respectively.

For a vector $x \in \mathbb{R}^{n}$, we will denote by $\|\cdot\|_{1}$ the $l_{1}$-norm, which is defined as $\|x\|_{1}=\sum_{i}^{n}\left|x_{i}\right|$, and denote by $\|\cdot\|_{2}$ the $l_{2}$-norm, which is defined as $\|x\|_{2}=\sqrt{\sum_{i}^{n} x_{i}^{2}}$. We use $\tilde{O}, \tilde{\Omega}$ instead of $O, \Omega$ when we neglect factors of logarithmic order in the parameters of the problem $(n, t, \epsilon)$. We denote by $\operatorname{Poi}(\lambda)$ the Poisson distribution with parameter $\lambda \in \mathbb{N}$.

\subsection{CDVV CDVV14 Estimator}

The idea behind the estimator from CDVV14 is similar to estimation using collisions $\mathrm{BFR}^{+} 13$, except it assumes Poisson sampling for getting some independence that simplifies the analysis, and therefore needs some corrective terms to shift the mean so that the estimation is unbiased. It is defined by first drawing a number $M \sim \operatorname{Poi}(\lambda)$, where $\lambda$ is some parameter high enough, and then taking $M$ arbitrary samples on each side and computing using the occurrence vectors $X, Y$ of those samples:

$$
\begin{aligned}
Z & =\frac{\sqrt{\sum_{i}\left(\left(X_{i}-Y_{i}\right)^{2}-X_{i}-Y_{i}\right)}}{M} \\
& =\frac{\sqrt{\sum_{i}\left(X_{i}-Y_{i}\right)^{2}-2 M}}{M} .
\end{aligned}
$$

\subsection{Classical Protocol}

We now describe the protocol from [AMN19] for the case of small $l_{2}$-norm.

The main idea behind the protocol is based on the CDVV estimator described in the previous subsection, combined with a reduction from $l_{1}$-distance estimation to $l_{2}$-distance estimation. Considering 
as well the errors of the estimator, after rescaling and shifting from $Z$, Andoni, Malkin and Nosatzki found that it is enough to compare some approximation of the term $\Delta=\|X-Y\|_{2}^{2}$ to some threshold $\tau$ to distinguish the two cases. As discussed in the introduction, the original protocol from [AMN19] has a communication step to recast the probability distributions into ones with smaller $l_{2}$-norms. Since this step is not necessary for the case of distributions with small $l_{2}$-norms, we omit it in the following description.

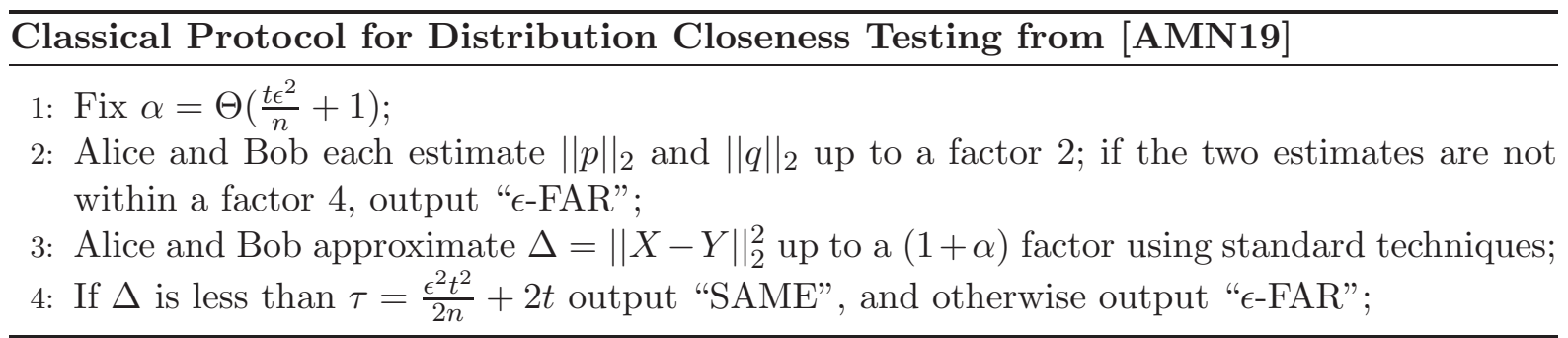

The analysis from AMN19, in particular Lemma 6, shows the correctness of the protocol. More precisely, the following statement can be obtained for the case of input distributions with low $l_{2}$-norms.

Theorem 3. AMN19] There exists an absolute constant $\gamma_{0}$ such that the following holds: for any input distributions $p$ and $q$ such that $\min \left(\|p\|_{2},\|q\|_{2}\right) \leq \gamma_{0} t \epsilon^{2} / n$, the above protocol correctly distinguish between the case $p=q$ and the case $\|p-q\|_{1} \geq \epsilon$ with probability at least $2 / 3$. bits.

The communication complexity is dominated by the third step, which requires $\tilde{O}\left(1 / \alpha^{2}\right)=\tilde{O}\left(n^{2} /\left(t^{2} \epsilon^{4}\right)+1\right)$

\section{Quantum Protocol}

In this section we describe our quantum protocol for the problem $2 \mathrm{PCT}_{n, t, \epsilon}^{\gamma}$, and prove Theorem 1

\subsection{Description of the Whole Protocol}

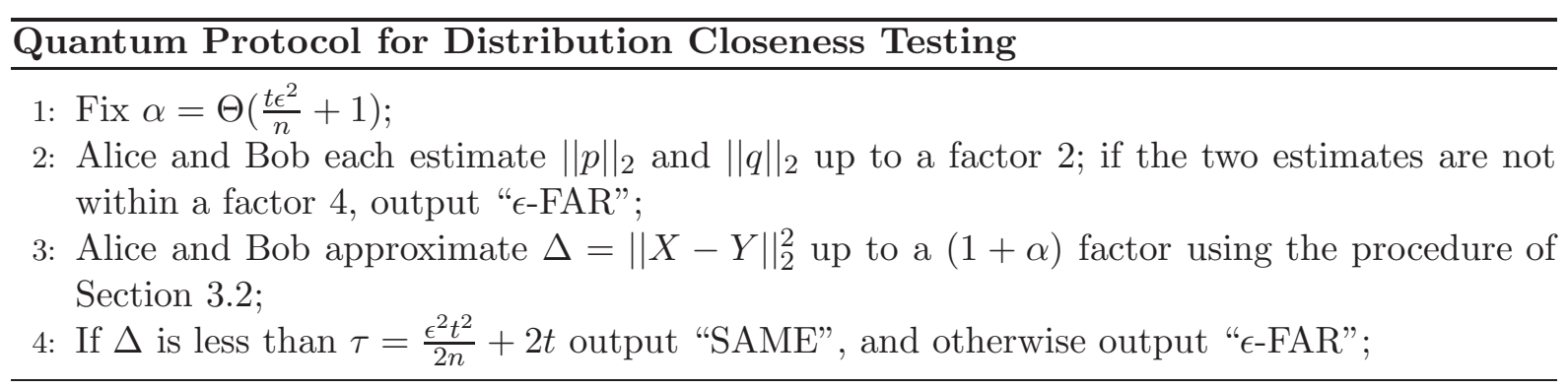

The communication complexity is again dominated by the third step, which requires only $\tilde{O}(1 / \alpha)=$ $\tilde{O}\left(n /\left(t \epsilon^{2}\right)+1\right)$ qubits, as described in the next subsection. The correctness is guaranteed by the analysis of Theorem 3. since the quantum protocol performs identical calculations as the classical protocol. This proves Theorem 1 .

\subsection{Montanaro Approximation}

The classical protocol AMN19] uses standard techniques, such as the AMS algorithm [AMS99, in order to approximate $\Delta=\|X-Y\|_{2}^{2}$. The AMS algorithm uses a family $\mathcal{H}$ of $O\left(n^{2}\right)$ hash functions $h_{i}:[n] \rightarrow\{-1,+1\}$ that are 4 -wise independent. Given a list of numbers $l=\left(l_{1} \ldots, l_{n}\right)$, the AMS algorithm gives an estimate of $\|l\|_{2}^{2}$ by computing random estimates $f(i)$ with the following subroutine many times and taking the median of the results 2

\footnotetext{
${ }^{2}$ The idea behind the AMS algorithm is that by developing the square, the "crossed" product terms' influence should vanish, i.e., $\mathbb{E}\left[h_{i}(j) l_{j} \cdot h_{i}\left(j^{\prime}\right) l_{j^{\prime}}\right]=0$ for $j \neq j^{\prime}$ because then $\mathbb{E}\left[h_{i}(j) \cdot h_{i}\left(j^{\prime}\right)\right]=0$, while the terms $h_{i}(j) l_{j} \cdot h_{i}(j) l_{j}=l_{j}^{2}$ will always stay.
} 


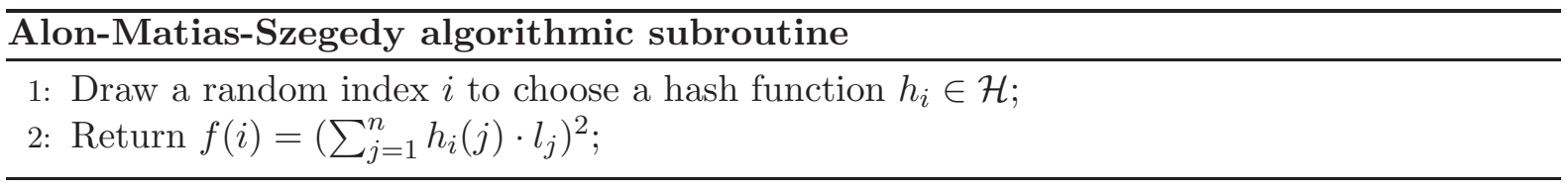

In the classical setting, this subroutine has to be repeated $\tilde{O}\left(1 / \alpha^{2}\right)$ times to get a $(1+\alpha)$-approximation.

Montanaro showed how to achieve the same approximation quantumly using this subroutine only $\tilde{O}(1 / \alpha)$ times (see Theorems 12 and 14 in Mon16]). The subroutine, however, needs to be called in superposition, i.e., Montanaro's approach requires a quantum oracle $O_{f}$ that performs the following map:

$$
O_{f}:|i\rangle|y\rangle \rightarrow|i\rangle|y+f(i)\rangle .
$$

It also requires access to its inverse $O_{f}^{-1}$.

In our communication setting, we want to use this approach with $l:=X-Y$. A difficulty is that the data is split between the two parties: only Alice knows $X$ and only Bob knows $Y$. We now explain how to overcome this difficulty. For a particular index $i$, Alice can compute $\sigma_{a}(i)=\sum_{j=1}^{n} h_{i}(j) X_{j}$, and then transmit it to Bob. Bob can similarly do his own computation $\sigma_{b}(i)=\sum_{j=1}^{n} h_{i}(j) Y_{j}$, then subtract $\sigma_{a}(i)$ and square in order to get

$$
\left(\sigma_{a}(i)-\sigma_{b}(i)\right)^{2}=\left(\sum_{j=1}^{n} h_{i}(j) \cdot\left(X_{j}-Y_{j}\right)\right)^{2}=f(i) .
$$

We describe below our implementation of the oracle $O_{f}$, based on these ideas. In the following description, Bob's input is the quantum state $|i\rangle_{I}|y\rangle_{Y}$ for some bit-strings $i$ and $y$, where $I$ and $Y$ are two quantum registers.

\section{Protocol for building the oracle $O_{f}$}

1: Bob maps $|i\rangle_{I}|y\rangle_{Y}$ to $|i\rangle_{I}|y\rangle_{Y}\left|\sigma_{b}(i)\right\rangle_{B}$ and sends register $I$ to Alice.

2: Alice creates another register $A$, computes the value $\sigma_{a}(i)$ in it, and sends the two registers $A$ and $I$ to Bob. At the end of this step, Bob thus owns

$$
|i\rangle_{I}|y\rangle_{Y}\left|\sigma_{a}(i)\right\rangle_{A}\left|\sigma_{b}(i)\right\rangle_{B}
$$

3: Bob performs the computation of Equation (2) using the arguments from $A$ and $B$, to get the state

$$
|i\rangle_{I}|y+f(i)\rangle_{Y}\left|\sigma_{a}(i)\right\rangle_{A}\left|\sigma_{b}(i)\right\rangle_{B}
$$

4: Bob erases the contents of register $B$, and sends registers $A$ and $I$ to Alice.

5: Alice erases the contents of register $A$ and sends back register $I$ to Bob.

Note that it is the linear property of the AMS computation (more precisely, Equation (2)) that allows the approximation to be computed even when the data are shared by several parties. Transmitting register $I$ requires $O(\log n)$ qubits. Transmitting register $A$ requires $O(\log t)$ qubits. The overall communication complexity of the oracle protocol is thus $O(\log n+\log t)$.

An implementation of the inverse $O_{f}^{-1}$ can be obtained similarly, by subtracting instead of adding the value of $f(i)$ at Step 3. We can thus apply Montanaro's algorithm [Mon16, simply by replacing each oracle query in Montanaro's algorithm by our distributed implementation of $O_{f}\left(\right.$ or $\left.O_{f}^{-1}\right)$. This enables us to obtain, with high probability, an $(1+\alpha)$-approximation of $\|l\|_{2}^{2}=\Delta$ using

$$
\tilde{O}((1 / \alpha)(\log n+\log t))=\tilde{O}(1 / \alpha)
$$

qubits of communication, as claimed.

\section{Quantum Lower Bound}

In this section we prove Theorem 2 , 


\subsection{Hamming Reduction}

For bit-strings $x, y \in\{0,1\}^{n}$, we denote by $x \cap y$ the set of indices where $x$ and $y$ both have a one, and write $|x \cap y|$ for its size. Note that for bit-strings the $l_{1}$-norm corresponds to Hamming weight, i.e., the number of ones in the string, and the $l_{1}$-distance corresponds to the Hamming distance.

In the classical communication setting, Andoni, Malkin and Nosatzki AMN19] proved a lower bound for some closeness testing problem by considering the following problem involving the Hamming distance of two binary strings.

Let $n \geq 1$ be a multiple of 4 . Let $\beta=\beta(n)=\sqrt{n / 32}$, and $\kappa>1$. With probability at least 0.9 , for $x, y \in\{0,1\}^{n}$ with $\|x\|_{1}=\|y\|_{1}=n / 2$, distinguish between the case where $\|x-y\|_{1}=n / 2$ versus $\|x-y\|_{1}-$ $n / 2 \in[\beta, \kappa \beta]$.

Notice that if $\|x\|_{1}=\|y\|_{1}=n / 2$ then the equality

$$
\|x-y\|_{1}=2(n / 2-|x \cap y|)=n-2|x \cap y|
$$

holds. The above problem then can be reformulated as the following communication problem that we call PromisedGHD $(n, \kappa)$ :

PromisedGHD $(n, \kappa)$
For $x, y \in\{0,1\}^{n}$, where $n$ is a multiple of 4 , with $\|x\|_{1}=\|y\|_{1}=n / 2$,
$\beta=\beta(n)=\sqrt{n / 32}$, and $\kappa>1$, with probability at least 0.9 distinguish
between $|x \cap y|=\frac{n}{4}$ and $|x \cap y| \in\left[\frac{n}{4}-\frac{\kappa \beta}{2}, \frac{n}{4}-\frac{\beta}{2}\right]$.

Particularly, the reduction of AMN19] only concerns the regime where $\kappa=O(\sqrt{\log n})$.

The main result of this subsection is:

Theorem 4. The quantum communication complexity of Promised $G H D(n, \kappa)$ is $\Omega(\sqrt{n})$.

In order to prove Theorem 4, we will consider a similar problem defined on smaller inputs. More precisely, define the following problem $\operatorname{SmallPGHD}\left(n^{\prime}, g\right)$ :

$\operatorname{SmallPGHD}\left(n^{\prime}, g\right)$
For $x^{\prime}, y^{\prime} \in\{0,1\}^{n^{\prime}}$ where $n^{\prime}$ is a multiple of 4 , with $\left\|x^{\prime}\right\|_{1}=\left\|y^{\prime}\right\|_{1}=$
$n^{\prime} / 2$, with probability at least $2 / 3$ distinguish between $\left|x^{\prime} \cap y^{\prime}\right|=n^{\prime} / 4$
and $\left|x^{\prime} \cap y^{\prime}\right| \in\left[n^{\prime} / 4-g, n^{\prime} / 4-1\right]$

We first show a reduction from $\operatorname{SmallPGHD}\left(n^{\prime}, g\right)$ to $\operatorname{PromisedGHD}(n, \kappa)$ for appropriate parameters.

Lemma 1. For any $g \leq \frac{\kappa}{2 \sqrt{32}}, \operatorname{SmallPGHD}\left(n^{\prime}, g\right)$ reduces to PromisedGHD $\left(n^{\prime 2}, \kappa\right)$.

Proof. Assume $x^{\prime}, y^{\prime}$ are inputs of size $n^{\prime}$ to the $\operatorname{SmallPGHD}\left(n^{\prime}, g\right)$ problem. By repeating them $n^{\prime}$ times in a padding fashion, we build inputs $x, y$ for the PromisedGHD $(n, \kappa)$ problem where $n=n^{\prime 2}$ and therefore $\beta=\frac{n^{\prime}}{\sqrt{32}}$. If $\left|x^{\prime} \cap y^{\prime}\right|=n^{\prime} / 4$, we have $|x \cap y|=n^{\prime 2} / 4=n / 4$. Otherwise, if $\left|x^{\prime} \cap y^{\prime}\right| \in\left[\frac{n^{\prime}}{4}-g, \frac{n^{\prime}}{4}-1\right]$, we have $|x \cap y| \in\left[\frac{n}{4}-\frac{\kappa \beta}{2}, \frac{n}{4}-\frac{\beta}{2}\right]$ as long as $g \leq \frac{\kappa}{2 \sqrt{32}}$. Indeed, for the upper part of the interval:

$$
\frac{n}{4}-\frac{\beta}{2}=\frac{n^{\prime 2}}{4}-\frac{n^{\prime}}{2 \sqrt{32}} \geq n^{\prime}\left(\frac{n^{\prime}}{4}-1\right)
$$

while for the lower part, we need:

$$
\frac{n}{4}-\frac{\kappa \beta}{2}=\frac{n^{\prime 2}}{4}-\frac{\kappa n^{\prime}}{2 \sqrt{32}} \leq n^{\prime}\left(\frac{n^{\prime}}{4}-g\right)
$$

which is equivalent to the bound on $g$ mentioned just above.

The hardness of $\operatorname{SmallPGHD}\left(n^{\prime}, g\right)$ will follow from the hardness of a more restricted problem stated in Theorem 5. The latter theorem is the main technical contribution of this section, and we devote Section 4.2 to its proof. 


\subsection{Main Technical Result}

We will model partial functions as mappings $f: X \rightarrow \mathbb{R} \cup\{*\}$, where $X$ is a finite set and the range element $*$ represents undefined output. We let $\operatorname{dom} f=\{x: f(x) \neq *\}$. It will be convenient here to represent the Boolean values "true" and "false" by -1 and +1 , respectively. This departure from the classical representation (of using 0 and 1) has no effect on quantum communication complexity. For a communication problem $F: X \times Y \rightarrow\{-1,+1, *\}$, we let $Q_{\epsilon}^{*}(F)$ denote the $\epsilon$-error quantum communication complexity of $F$ with arbitrary prior entanglement. Note that an $\epsilon$-error protocol for $F$ is allowed to behave arbitrarily on inputs outside $\operatorname{dom} F$.

We are now ready to prove the main technical result used in the proof of Theorem 2

Theorem 5. Let $n$ be an integer divisible by 4 . Consider the partial communication problem $F_{n}:\{0,1\}^{n} \times$ $\{0,1\}^{n} \rightarrow\{-1,+1, *\}$ given by

$$
F_{n}(x, y)= \begin{cases}-1 & \text { if }|x|=|y|=n / 2 \text { and }|x \cap y|=n / 4 \\ +1 & \text { if }|x|=|y|=n / 2 \text { and }|x \cap y|=n / 4-1 \\ * & \text { otherwise. }\end{cases}
$$

Then $Q_{1 / 3}^{*}\left(F_{n}\right)=\Omega(n)$.

The remaining part of this section is devoted to the proof of this theorem. We start by reviewing relevant background on the pattern matrix method She11 for quantum communication lower bounds. Let $k$ and $n$ be positive integers, where $k<n$ and $k \mid n$. Partition $[n]$ into $k$ contiguous blocks, each with $n / k$ elements:

$$
[n]=\left\{1,2, \ldots, \frac{n}{k}\right\} \cup\left\{\frac{n}{k}+1, \ldots, \frac{2 n}{k}\right\} \cup \cdots \cup\left\{\frac{(k-1) n}{k}+1, \ldots, n\right\}
$$

Let $\mathcal{V}(n, k)$ denote the family of subsets $V \subseteq[n]$ that have exactly one element in each of these blocks (in particular, $|V|=k$ ). Clearly, $|\mathcal{V}(n, k)|=(n / k)^{k}$. For a bit string $x \in\{0,1\}^{n}$ and a set $V \in \mathcal{V}(n, k)$, define the projection of $x$ onto $V$ by $\left.x\right|_{V}=\left(x_{i_{1}}, x_{i_{2}}, \ldots, x_{i_{k}}\right) \in\{0,1\}^{k}$, where $i_{1}<i_{2}<\cdots<i_{k}$ are the elements of $V$. For $\phi:\{0,1\}^{k} \rightarrow \mathbb{R} \cup\{*\}$, the $(n, k, \phi)$-pattern matrix is the matrix $A$ given by

$$
A=\left[\phi\left(\left.x\right|_{V} \oplus w\right)\right]_{x \in\{0,1\}^{n},(V, w) \in \mathcal{V}(n, k) \times\{0,1\}^{k}} .
$$

In words, $A$ is the matrix of size $2^{n}$ by $(n / k)^{k} 2^{k}$ whose rows are indexed by strings $x \in\{0,1\}^{n}$, whose columns are indexed by pairs $(V, w) \in \mathcal{V}(n, k) \times\{0,1\}^{k}$, and whose entries are given by $A_{x,(V, w)}=$ $\phi\left(\left.x\right|_{V} \oplus w\right)$.

The pattern matrix method gives a lower bound on the quantum communication complexity of a pattern matrix in terms of the approximate degree of its generating function. We now define this notion formally. Let $f: X \rightarrow \mathbb{R}$ be given, for a finite subset $X \subset \mathbb{R}^{n}$. The $\epsilon$-approximate degree of $f$, denoted $\operatorname{deg}_{\epsilon}(f)$, is the least degree of a real polynomial $\pi$ such that $|f(x)-\pi(x)| \leq \epsilon$ for all $x \in X$. One generalizes this definition to partial functions $f: X \rightarrow \mathbb{R} \cup\{*\}$ by letting $\operatorname{deg}_{\epsilon}(f)$ be the least degree of a real polynomial $\pi$ with

$$
\begin{array}{ll}
|f(x)-\pi(x)| \leq \epsilon, & x \in \operatorname{dom} f \\
|\pi(x)| \leq 1+\epsilon, & x \in X \backslash \operatorname{dom} f
\end{array}
$$

We will need the following version of the pattern matrix method for quantum lower bounds.

Theorem 6. Let $F$ be the $(n, k, f)$-pattern matrix, where $f:\{0,1\}^{k} \rightarrow\{-1,+1, *\}$ is given. Then for every $\epsilon \in[0,1)$ and every $\delta<\epsilon / 2$,

$$
Q_{\delta}^{*}(F) \geq \frac{1}{4} \operatorname{deg}_{\epsilon}(f) \log \left(\frac{n}{k}\right)-\frac{1}{2} \log \left(\frac{3}{\epsilon-2 \delta}\right) .
$$

Theorem [ 6 is a generalization of the original pattern matrix method of She1] to partial functions. For the reader's convenience, we give a detailed proof of Theorem 6 in Appendix A 
Proof of Theorem 5. The communication complexity of $F_{n}$ is monotone in $n$, due to $F_{n}(x, y)=$ $F_{n+4}(x 0011, y 0101)$. As a result, it suffices to prove the theorem for $n$ divisible by 3 . Under this divisibility assumption, define $k=n / 6$ and consider the function $\operatorname{PMAJ}_{k}:\{0,1\}^{k} \rightarrow\{-1,+1, *\}$ given by

$$
\operatorname{PMAJ}_{k}(x)= \begin{cases}-1 & \text { if }|x|=k / 2 \\ +1 & \text { if }|x|=k / 2-1 \\ * & \text { otherwise }\end{cases}
$$

Let $P$ be the $\left(2 k, k, \mathrm{PMAJ}_{k}\right)$-pattern matrix. It is a well-known fact Pat92, BT15 that $\operatorname{deg}_{1 / 3}\left(\mathrm{PMAJ}_{k}\right)=$ $\Omega(k)$. As a result, Theorem 6 implies that $Q_{1 / 7}^{*}(P)=\Omega(k)$ and hence also $Q_{1 / 3}^{*}(P)=\Omega(k)$.

Writing $P=\left[\mathrm{PMAJ}_{k}\left(\left.\left(x_{1} \bar{x}_{1} x_{2} \bar{x}_{2} \ldots x_{2 k} \bar{x}_{2 k}\right)\right|_{V}\right)\right]_{x \in\{0,1\}^{2 k}, V \in \mathcal{V}(4 k, k)}$ makes it clear that $P$ is a restriction of the more general communication problem $G:\{0,1\}^{4 k} \times\{0,1\}^{4 k} \rightarrow\{-1,+1, *\}$ defined by

$$
G(x, y)= \begin{cases}-1 & \text { if }|x|=2 k,|y|=k, \text { and }|x \cap y|=k / 2 \\ +1 & \text { if }|x|=2 k,|y|=k, \text { and }|x \cap y|=k / 2-1 \\ * & \text { otherwise. }\end{cases}
$$

As a result, $Q_{1 / 3}^{*}(G) \geq Q_{1 / 3}^{*}(P)=\Omega(k)$. This in turn implies that $Q_{1 / 3}^{*}\left(F_{n}\right)=\Omega(k)$ because $G(x, y)=$ $F_{n}\left(x 1^{k} 0^{k}, y 1^{2 k}\right)$.

\subsection{Closeness Testing Reduction}

In this subsection we explain how the lower bound on the quantum communication complexity of PromisedGHD $(n, \kappa)$ (Theorem 4) implies Theorem 2

Proof of Theorem 2 In AMN19, Andoni, Malkin and Nosatzki show a reduction from the problem PromisedGHD $(m, \kappa)$ to the problem $2 \mathrm{PCT}_{n, t, 1 / 2}$ with parameters $m=\frac{n^{2}}{t^{2} \log ^{3} n}$ and $\kappa=O(\sqrt{\log n}) 3$ Theorem 4 thus gives us the claimed lower bound. In the remaining of the proof, we show that the distributions used to prove the lower bound have low $l_{2}$-norm.

The input distributions of $2 \mathrm{PCT}_{n, t, 1 / 2}$ used in the reduction shown in AMN19, which we will denote $a$ and $b$, are of the following form: half of the mass is uniformly distributed on $d=n / 10$ elements, and the other half of the mass on $l=C_{0} \cdot t \cdot \log n$ other elements, where $C_{0}$ is some constant. Therefore:

$$
\begin{aligned}
\|a\|_{2}=\|b\|_{2} & =\sqrt{d\left(\frac{1}{2 d}\right)^{2}+l\left(\frac{1}{2 l}\right)^{2}} \\
& =\frac{1}{2} \sqrt{\frac{1}{d}+\frac{1}{l}} \\
& =\frac{1}{2} \sqrt{\frac{10}{n}+\frac{1}{C_{0} t \log n}} \\
& \leq \frac{1}{2} \sqrt{\left(10+\frac{1}{C_{0}}\right) \frac{1}{t \log n}},
\end{aligned}
$$

since $\frac{1}{n} \leq \frac{1}{t \log n}$ because $t \leq \frac{n}{\log ^{c} n}$.

Define $\gamma_{L W}$ as the smallest $\gamma$ such that $\min \left(\|a\|_{2},\|b\|_{2}\right) \leq \gamma t \epsilon^{2} / n$ holds. The above calculations show that

$$
\gamma_{L W}=\frac{\|a\|_{2} n}{t \epsilon^{2}} \leq \frac{1}{2} \sqrt{10+\frac{1}{C_{0}}} \frac{n}{t \epsilon^{2} \sqrt{t \log n}} \leq \frac{1}{2 C^{3 / 2}} \sqrt{10+\frac{1}{C_{0}}} \frac{1}{\sqrt{\log n}}
$$

because $t \geq C n^{2 / 3} \cdot \epsilon^{-4 / 3}$ by (1). Thus $\gamma_{L W}=O(1 / \sqrt{\log n})$. This concludes the proof.

Acknowledgements. Guillaume Malod was partially supported by a JSPS Invitational Fellowships for Research in Japan. Aleksandrs Belovs is supported by the ERDF project number 1.1.1.2/I/16/113.

\footnotetext{
${ }^{3}$ The reduction is actually stated, in Theorem 9 in AMN19, as a reduction from PromisedGHD $(m, \kappa)$ to the variant of $2 \mathrm{PCT}_{n, t, 1 / 2}$ where the number of samples is Poi $(t)$ instead of exactly $t$. Nevertheless the Poisson version easily reduces to the original problem with $10 t$ number of samples if we allow some extra error, since by Chebyshev's inequality the probability that the Poisson version gives more than $10 t$ samples is less than $1 / 81$.
} 
Arturo Castellanos is grateful to Shin-ichi Minato for his support, and also to MEXT. Alexander A. Sherstov was supported by NSF grant CCF-1814947. François Le Gall was supported by JSPS KAKENHI grants Nos. JP16H01705, JP19H04066, JP20H00579, JP20H04139 and by the MEXT Quantum Leap Flagship Program (MEXT Q-LEAP) grant No. JPMXS0118067394.

\section{References}

[AdW17] Srinivasan Arunachalam and Ronald de Wolf. Guest column: A survey of quantum learning theory. SIGACT News, 48(2):41-67, 2017.

[AMN19] Alexandr Andoni, Tal Malkin, and Negev Shekel Nosatzki. Two party distribution testing: Communication and security. In Proceedings of the 46th International Colloquium on Automata, Languages, and Programming (ICALP 2019), volume 132 of LIPIcs, pages 15:1$15: 16,2019$.

[AMS99] Noga Alon, Yossi Matias, and Mario Szegedy. The space complexity of approximating the frequency moments. Journal of Computer and System Sciences, 58(1):137-147, 1999.

$\left[\mathrm{BFR}^{+} 00\right]$ Tugkan Batu, Lance Fortnow, Ronitt Rubinfeld, Warren D. Smith, and Patrick White. Testing that distributions are close. In 41st Annual Symposium on Foundations of Computer Science, FOCS 2000, pages 259-269. IEEE Computer Society, 2000.

$\left[\mathrm{BFR}^{+} 13\right]$ Tugkan Batu, Lance Fortnow, Ronitt Rubinfeld, Warren D. Smith, and Patrick White. Testing closeness of discrete distributions. J. ACM, 60(1):4:1-4:25, 2013.

[Bra04] Gilles Brassard. Quantum communication complexity: A survey. In 34th IEEE International Symposium on Multiple-Valued Logic (ISMVL 2004), 19-22 May 2004, Toronto, Canada, page 56. IEEE Computer Society, 2004.

[BT15] Mark Bun and Justin Thaler. Dual lower bounds for approximate degree and MarkovBernstein inequalities. Inf. Comput., 243:2-25, 2015.

[Can15] Clément L. Canonne. A survey on distribution testing: Your data is big. but is it blue? Electronic Colloquium on Computational Complexity (ECCC), 22:63, 2015.

[CDVV14] Siu-on Chan, Ilias Diakonikolas, Paul Valiant, and Gregory Valiant. Optimal algorithms for testing closeness of discrete distributions. In Proceedings of the Twenty-Fifth Annual ACM-SIAM Symposium on Discrete Algorithms (SODA 2014), pages 1193-1203, 2014.

[DK16] Ilias Diakonikolas and Daniel M. Kane. A new approach for testing properties of discrete distributions. In Irit Dinur, editor, Proceedings of the IEEE 5\%th Annual Symposium on Foundations of Computer Science (FOCS 2016), pages 685-694. IEEE Computer Society, 2016.

[dW02] Ronald de Wolf. Quantum communication and complexity. Theor. Comput. Sci., 287(1):337$353,2002$.

[Gol17] Oded Goldreich. Introduction to Property Testing. Cambridge University Press, 2017.

[GR11] Oded Goldreich and Dana Ron. On testing expansion in bounded-degree graphs. In Oded Goldreich, editor, Studies in Complexity and Cryptography. Miscellanea on the Interplay between Randomness and Computation, volume 6650 of Lecture Notes in Computer Science, pages 68-75. Springer, 2011.

[KN97] Eyal Kushilevitz and Noam Nisan. Communication complexity. Cambridge University Press, 1997.

[MdW16] Ashley Montanaro and Ronald de Wolf. A survey of quantum property testing. Theory of Computing, Graduate Surveys, 7:1-81, 2016.

[Mon16] Ashley Montanaro. The quantum complexity of approximating the frequency moments. Quantum Information \& Computation, 16(13\&14):1169-1190, 2016.

[Pat92] Ramamohan Paturi. On the degree of polynomials that approximate symmetric Boolean functions. In Proceedings of the Twenty-Fourth Annual ACM Symposium on Theory of Computing (STOC), pages 468-474, 1992. 
[She11] Alexander A. Sherstov. The pattern matrix method. SIAM Journal on Computing, 40(6):1969-2000, 2011.

[She12] Alexander A. Sherstov. Strong direct product theorems for quantum communication and query complexity. SIAM J. Comput., 41(5):1122-1165, 2012.

\section{A The Pattern Matrix Method for Partial Functions}

The purpose of this appendix is to provide a detailed proof of Theorem 6 for partial functions. Our proof closely follows the original proof of the pattern matrix method in [She11, developed there for total functions.

We start by recalling the Fourier transform for functions $f:\{0,1\}^{n} \rightarrow \mathbb{R}$. For $S \subseteq\{1,2, \ldots, n\}$, define $\chi_{S}:\{0,1\}^{n} \rightarrow\{-1,+1\}$ by $\chi_{S}(x)=(-1)^{\sum_{i \in S} x_{i}}$. Then every function $f:\{0,1\}^{n} \rightarrow \mathbb{R}$ has a unique representation of the form

$$
f=\sum_{S \subseteq\{1,2, \ldots, n\}} \hat{f}(S) \chi_{S},
$$

where $\hat{f}(S)=2^{-n} \sum_{x \in\{0,1\}^{n}} f(x) \chi_{S}(x)$. The reals $\hat{f}(S)$ are called the Fourier coefficients of $f$.

For a real matrix $A$, we let $\|A\|_{1}$ denote the sum of the absolute values of the entries of $A$. We let $\|A\|$ denote the spectral norm of $A$. Recall that $\|A\|=\max _{x:\|x\|_{2}=1}\|A x\|_{2}$. The following theorem [She11, Theorem 4.3] determines the spectral norm of a pattern matrix in terms of the Fourier spectrum of its generating function.

Theorem 7. Let $\phi:\{0,1\}^{k} \rightarrow \mathbb{R}$ be given. Let $A$ be the $(n, k, \phi)$-pattern matrix. Then

$$
\|A\|=\sqrt{2^{n+k}\left(\frac{n}{k}\right)^{k}} \max _{S \subseteq[k]}\left\{|\hat{\phi}(S)|\left(\frac{k}{n}\right)^{|S| / 2}\right\} .
$$

We will also need the following dual characterization of approximate degree of partial functions, analogous to the dual characterization for total functions used in [She11.

Theorem 8. Let $f:\{0,1\}^{n} \rightarrow \mathbb{R} \cup\{*\}$ be a given function, $d \geq 0$ an integer. Then $\operatorname{deg}_{\epsilon}(f)>d$ if and only if there exists $\psi:\{0,1\}^{n} \rightarrow \mathbb{R}$ such that

$$
\sum_{x \in \operatorname{dom} f} f(x) \psi(x)-\sum_{x \notin \operatorname{dom} f}|\psi(x)|-\epsilon\|\psi\|_{1}>0,
$$

and $\hat{\psi}(S)=0$ for $|S| \leq d$.

Theorem 8 follows from linear programming duality; see [She11, She12] for details.

Next, we derive a version of the generalized discrepancy method for partial functions, by adapting the analogous proof in She11, Theorem 2.8] for total functions.

Theorem 9. Let $X, Y$ be finite sets and $F: X \times Y \rightarrow\{-1,+1, *\}$ a given function. Let $\Psi=\left[\Psi_{x y}\right]_{x \in X, y \in Y}$ be any real matrix with $\|\Psi\|_{1}=1$. Then for each $\epsilon>0$,

$$
4^{Q_{\epsilon}^{*}(F)} \geq \frac{1}{3\|\Psi\| \sqrt{|X||Y|}}\left(\sum_{(x, y) \in \operatorname{dom} F} \Psi_{x, y} F(x, y)-\sum_{(x, y) \notin \operatorname{dom} F}\left|\Psi_{x, y}\right|-2 \epsilon\right) .
$$

Proof. Let $P$ be a quantum protocol with prior entanglement that computes $F$ with error $\epsilon$ and $\operatorname{cost} C$. Let $\Pi$ be the matrix of acceptance probabilities of $P$, so that $\Pi_{x, y}$ is the probability that $P$ accepts the input $(x, y)$. It is shown in the proof of [She11, Theorem 2.8] that

$$
\sum_{x \in X} \sum_{y \in Y} \Psi_{x, y}\left(1-2 \Pi_{x, y}\right) \leq\|\Psi\|\left(2 \cdot 4^{C}+1\right) \sqrt{|X||Y|} .
$$


Now observe that $1-2 \Pi_{x, y}$ ranges in $[F(x, y)-2 \epsilon, F(x, y)+2 \epsilon]$ on $\operatorname{dom} F$, and is bounded in absolute value by 1 otherwise. This gives

$$
\begin{aligned}
\sum_{x \in X} \sum_{y \in Y} \Psi_{x, y}\left(1-2 \Pi_{x, y}\right) & \\
& \geq \sum_{(x, y) \in \operatorname{dom} F}\left(\Psi_{x, y} F(x, y)-2 \epsilon\left|\Psi_{x, y}\right|\right)-\sum_{(x, y) \notin \operatorname{dom} F}\left|\Psi_{x, y}\right| \\
& \geq \sum_{(x, y) \in \operatorname{dom} F} \Psi_{x, y} F(x, y)-2 \epsilon-\sum_{(x, y) \notin \operatorname{dom} F}\left|\Psi_{x, y}\right|,
\end{aligned}
$$

where the last step uses $\|\Psi\|_{1} \leq 1$. The theorem follows by comparing the upper bound (3) with the lower bound (41).

We are now in a position to prove Theorem [ which we restate here for the reader's convenience.

Theorem 10 (restatement of Theorem [6). Let $F$ be the $(n, k, f)$-pattern matrix, where $f:\{0,1\}^{k} \rightarrow$ $\{-1,+1, *\}$ is given. Then for every $\epsilon \in[0,1)$ and every $\delta<\epsilon / 2$,

$$
Q_{\delta}^{*}(F) \geq \frac{1}{4} \operatorname{deg}_{\epsilon}(f) \log \left(\frac{n}{k}\right)-\frac{1}{2} \log \left(\frac{3}{\epsilon-2 \delta}\right) .
$$

Proof. Let $d=\operatorname{deg}_{\epsilon}(f) \geq 1$. By Theorem 8 , there is a function $\psi:\{0,1\}^{k} \rightarrow \mathbb{R}$ such that:

$$
\begin{aligned}
& \hat{\psi}(S)=0 \\
& \sum_{z \in\{0,1\}^{k}}|\psi(z)|=1, \quad(|S|<d), \\
& \sum_{z \in \operatorname{dom} f} f(z) \psi(z)-\sum_{z \notin \operatorname{dom} f}|\psi(z)|>\epsilon .
\end{aligned}
$$

Let $\Psi$ be the $\left(n, k, 2^{-n}(n / k)^{-k} \psi\right)$-pattern matrix. Then (7) and (8) show that

$$
\sum_{(x, y) \in \operatorname{dom} F}^{\|\Psi\|_{1}=1,} F_{x, y} \Psi_{x, y}-\sum_{(x, y) \notin \operatorname{dom} F}\left|\Psi_{x, y}\right|>\epsilon .
$$

Our last task is to calculate $\|\Psi\|$. It follows from (17) that

$$
\max _{S \subseteq[k]}|\hat{\psi}(S)| \leq 2^{-k}
$$

Theorem 7 yields, in view of (6) and (11):

$$
\|\Psi\| \leq\left(\frac{k}{n}\right)^{d / 2}\left(2^{n+k}\left(\frac{n}{k}\right)^{k}\right)^{-1 / 2} .
$$

Now (5) follows from (9), (10), (12), and Theorem 9. 